\title{
Liver resection for non-colorectal metastases
}

\author{
Christoph Schwarz - Klaus Kaczirek - Martin Bodingbauer
}

Received: 11 January 2018 / Accepted: 9 April 2018 / Published online: 25 April 2018

(C) The Author(s) 2018

\begin{abstract}
Summary Whereas liver resection for colorectal metastasis has become standard of care, hepatectomy in patients with non-colorectal metastases remains controversial, mainly due to a heterogeneous tumor biology and missing data from prospective trials. This review aims at giving an overview about the indications and limits of liver surgery in patients with an advanced disease of a non-colorectal malignancy. Even though prospective trials are largely missing, results from retrospective studies indicate a survival benefit for liver resection in selected patients. Thus, in metastasized patients, treatment strategies should be developed in a multidisciplinary tumor board including an experienced liver surgeon.
\end{abstract}

Keywords Liver metastases - Surgical technical improvement - Liver resection - Oncological prognosis . Oncological surgical treatment

\section{Introduction}

The liver is a frequent site for metastases of various kinds of malignancies. Whereas historically, metastasized malignancies have had a dismal outcome and limited treatment options, refinement of surgical techniques and advances in systemic and locoregional oncological therapy have pushed the indications for liver resection (LR) towards a more aggressive approach, where hepatectomy is still a potential option even in patients with advanced cancer [1].

Whereas liver resection for colorectal metastasis has been widely accepted, resulting in a substantially improved patient survival, hepatectomy for non-col-

C. Schwarz $\cdot$ K. Kaczirek $\cdot$ M. Bodingbauer, MD $(\bowtie)$

Department of Surgery, Medical University of Vienna,

Währinger Gürtel 18-20, 1090 Vienna, Austria

martin.bodingbauer@meduniwien.ac.at orectal metastases remains controversial and is still under debate, which is mainly attributed to heterogeneous tumor subtypes.

This review aims at giving an overview about the indications and limits of LR in non-colorectal liver metastasis.

\section{Breast cancer (BRE)}

After metastasizing in lymph nodes and lungs, the liver represents the third most common site for metastases of breast cancer [2]. Even though treatment strategies of metastasized breast cancer include systemic therapy, the use of liver resection has gained more and more acknowledgement. Only small retrospective studies investigating the impact of LR on survival for breast cancer metastasis have been published. However, a survival benefit has been suggested throughout the studies [3, 4].

Recently it has been shown in a cost-effectiveness study that liver resection for breast cancer metastases is cost-effective compared to systemic therapy alone, mainly in estrogen receptor-positive patients [5]. Currently, 5 -year overall survival is reported to range between 27 and 53\%, depending on patient- and tumorrelated factors [6]. Prognostic factors determining the outcome are response to preoperative chemotherapy, resection margin, and re-liver resection [4] or hormone receptor status $[7,8]$. Especially patients with estrogen receptor-positive status might benefit from liver resection.

Mariani et al. proposed the indication for LR to be resectable liver metastasis ( $\leq 4$ metastases), stable disease or disease responding on medical treatment, good patient performance status, and the only allowable extrahepatic disease being stable bone metastasis [9]. 


\section{Gastrointestinal (GI)}

Due to the venous drainage via the portal vein, most gastrointestinal malignancies metastasize primarily to the liver. GI malignancies mainly include esophageal cancer, gastric adenocarcinoma, and gastrointestinal stromal tumors (GIST).

Liver metastases in patients with gastric cancer are diagnosed in up to 2-14\% synchronously [10] and in $37 \%$ metachronously after gastric resection [11]. A recent meta-analysis suggests improved survival rates following liver resection for gastric adenocarcinoma (hazard ratio, HR: 0.50; [12]). Negative risk factors include multiple liver metastasis $[13,14]$, the size of metastases, and serosal invasion of the primary gastric cancer [14]. Thus, currently, the resection of small numbers metastases is recommended in combination with perioperative chemotherapy [15].

GISTs are the most common mesenchymal tumors of the gastrointestinal tract and it is estimated that $47 \%$ are synchronously metastasized [16], with the liver being the most frequent site of metastasis [17]. After LR, 5-year survival rates range between 70 and $76 \%[18,19]$, with complete resection (R0) being a significant prognostic factor for long-term survival [19]. Notably, preoperative tyrosine kinase inhibitor (TKI) therapy is suggested to be connected with a benefit in survival [19-21]. Thus, LR should only be recommended in patients under perioperative TKI therapy who are responding on medical treatment.

Only small case series exist regarding LR for metastasis of esophageal cancer. Even though most studies suggest LR is feasible in selected patients, 5-year survival of patients after LR is less than $15 \%$, and therefore currently not generally recommended [18].

\section{Melanoma (MEL)}

There are two subgroups of melanoma: cutaneous and ocular melanoma, both with distinct pathological features. Because of the absence of lymphatic tissue in the uveal tract, ocular melanomas mainly metastasize hematogenously, with the liver being the main affected organ. In contrast, cutaneous melanoma rarely metastasizes to the liver but rather to the lungs, lymph nodes, or soft tissues [22]. From retrospective studies, 5 -year survival after LR is between 7 and $20 \%$, with a median survival of 14-28 months [6]. Notably, R0 resection should be the aim [23, 24].

Overall, studies suggest a survival benefit for surgery in resectable patients; however, LR should be performed with concomitant systemic therapy, as recurrence is common after hepatectomy.

\section{Pancreato-biliary (PB)}

Pancreatic ductal adenocarcinoma (PDAC) is one of the most fatal malignancies, with an increasing incidence over the past decades $[25,26]$. It has been suggested that the metastases of pancreatic cancer occur relatively late after the initial development of the primary tumor [27]. Due to limited systemic treatment options, it is generally not recommended to resect metastases of PDAC [28, 29]. However, some case series have suggested that liver resection might be a potential option in selected patients with synchronous or even metachronous disease [30, 31]. Five-year survival after LR for PDAC metastasis is low and ranges between 5.8 and $20 \%$ [18, 30, 32]. Thus, currently, LR for patients with metastasized PDAC should not be performed in all patients but rather only in patients with a good tumor biology (response to chemotherapy, long interval between primary resection and appearance of liver metastases in patients with metachronous disease).

\section{Genitourinary (GU)}

Genitourinary tumors mainly include renal cell carcinomas (RCC), gynecological carcinomas especially ovarian cancer, and testicular malignancies.

For patients with RCC undergoing LR, overall 5-year survival rates of $38-62 \%$ were reported, with a median survival of 33-142 months [33-35]. Risk factors for poor outcome are extrahepatic disease, a short disease-free interval [35], lymph node metastases, poor performance status, high-grade tumors [34], and a positive resection margin [33].

5 -year overall survival rates of $30-51 \%$ in patients with ovarian cancer were described in several studies with median survival times of 26-98 months [36, 37]. Risk factors for worse prognosis are a short interval from diagnosis of primary disease to metastasis [37], positive resection margins, pre-operative ascites, and bilobar disease [36].

Only limited data exist for hepatectomy for testicular carcinoma, but the combination of chemotherapy and liver surgery demonstrated improved outcome in patients who faced this treatment plan [38].

\section{Head and neck (HN)}

Head and neck cancers include oral, nasopharyngeal, and laryngeal malignancies. These cancers are most frequently squamous cell carcinomas. In general, these patients experience poor prognosis following LR, with 5-year survival rates of less than $15 \%$ [18]. Better outcome was reported for liver metastases from undifferentiated nasopharyngeal carcinomas with an overall survival rate of $40.2 \%$ [39].

\section{Thyroid cancer (TC)}

Papillary and follicular thyroid carcinomas are known as differentiated thyroid carcinomas (DTC) and are among the most curable of cancers. Liver metastases from DTC are exceptionally rare; only a few cases are reported in the literature, but surgical resection 
should always be considered as this offers the best chance for prolonged survival [40].

\section{Neuroendocrine tumors (NET)}

Neuroendocrine tumors often metastasize to the liver (50-75\%). Surgical resection and transplantation have been offered in a curative intent, but palliative cytoreductive surgery can also be considered for disease control. In only few patients $(7-15 \%)$ can a curative resection be realized [41]. The role of surgical intervention was studied to be the best approach for resectable liver metastases from NET in terms of prolonged survival and relief of symptoms, despite the high incidence of recurrence after surgery [42].

Hepatectomy has become a safe surgical procedure and resectability can more often be pushed to a further level also in advanced oncological states. In the context of a metastatic setting of an NET tumor, the surgical intention should be the removal of all NET metastases within the liver parenchyma with curative intent. Even in combination with non-resectable liver-directed treatments, including ablative techniques, transarterial chemoembolization (TACE), or internal radiation therapy (radioembolization). All these new treatments have been shown to be successful in slowing tumor progression and providing symptom relief $[43,44]$.

In general, well-differentiated, low- or intermediate-grade NETs have a relatively indolent behavior with slow progression and can be treated. Poorly differentiated tumors may exhibit highly aggressive behavior with rapid metastatic spread, and surgery therefore plays an insufficient treatment role in this setting.

Only a low level of recommendations exists to assist in the treatment of patients with neuroendocrine liver metastases. The efficacy of the NET management, either surgery alone or in combination with non-resectable treatments, remains unproven [45].

\section{Cancer of unknown primary (CUP)}

A cancer of unknown primary is often associated with an unfortunate outcome, because patients are usually treated with non-standardized therapy. LR of metastases of a CUP can improve prognosis and is reported with a 5-year survival of $38 \%$ [18].

\section{Discussion}

Liver surgery has become a valuable treatment option even for patients with advanced cancer. In combination with suitable neoadjuvant and adjuvant chemotherapy, local tumor control is the only chance to ensure long-term survival for patients with metastasized disease. While LR for colorectal or neuroendocrine metastasis has become standard of care today, hepatectomy for non-colorectal metastasis remains controversial, which is mainly attributed to a lack of prospective data due to a relatively low incidence, but also due to missing powerful chemotherapy options for most tumor types. However, the safety of hepatectomy has increased over the past decades [1] and oncological therapy has improved substantially. Therefore, aggressive treatment even of an advanced disease is gaining more and more acknowledgment.

Prospective trials are necessary to better characterize the role of liver resection embedded in a multidisciplinary treatment approach. Moreover, an adequate patient selection based on tumor biology and patientrelated factors is important to further improve outcome after LR.

Liver resection is a suitable treatment for non-colorectal cancer liver metastases. It can offer acceptable long-term survival, comparable to colorectal cancer metastases. Patients with controlled systemic disease benefit from hepatic resection and the treatment of liver metastases should be considered regardless of the primary tumor site. For individual patients with controlled systemic disease, hepatic resection can offer appropriate survival rates and should be considered regardless of the primary tumor site. It should be part of the oncological treatment.

Funding Open access funding provided by Medical University of Vienna.

Conflict of interest C. Schwarz, K. Kaczirek, and M. Bodingbauer MD declare that they have no competing interests.

Open Access This article is distributed under the terms of the Creative Commons Attribution 4.0 International License (http://creativecommons.org/licenses/by/4.0/), which permits unrestricted use, distribution, and reproduction in any medium, provided you give appropriate credit to the original author(s) and the source, provide a link to the Creative Commons license, and indicate if changes were made.

\section{References}

1. Clavien P, Petrowsky H, DeOliveira ML, Graf R. Medical progress: strategies for safer liver surgery and partial liver transplantation. NewEngl J Med. 2007;356:1545-59.

2. Disibio G, French SW. Metastatic patterns of cancers: results from a large autopsy study. Arch Pathol Lab Med. 2008;132:931-9.

3. Polistina F, Costantin G, Febbraro A, et al. Aggressive treatment for hepatic metastases from breast cancer: results from a single center. World J Surg. 2013;37:1322-32.

4. Adam R, Aloia T, Krissat J, et al. Is liver resection justified for patients with hepatic metastases from breast cancer? Ann Surg. 2006;244:897-907. discussion 907-898.

5. Spolverato G, Vitale A, Bagante F, et al. Liver resection for breast cancer liver metastases: a cost-utility analysis. Ann Surg. 2017;265:792-9.

6. Takemura N, Saiura A. Role of surgical resection for noncolorectal non-neuroendocrine liver metastases. World J Hepatol. 2017;9:242-51.

7. Elias D, Maisonnette F, Druet-Cabanac M, et al. An attempt to clarify indications for hepatectomy for liver metastases from breast cancer. Am J Surg. 2003;185:158-64. 
8. Abbott DE, Brouquet A, Mittendorf EA, et al. Resection of liver metastases from breast cancer: Estrogen receptor status and response to chemotherapy before metastasectomy define outcome. Surgery. 2012;151:710-6.

9. Mariani P, Servois V, De Rycke Y, et al. Liver metastases from breast cancer: Surgical resection or not? A case-matched control study in highly selected patients. Eur J Surg Oncol. 2013;39:1377-83.

10. SakamotoY, OhyamaS, YamamotoJ, etal. Surgical resection of liver metastases of gastric cancer: an analysis of a 17-year experience with 22 patients. Surgery. 2003;133:507-11.

11. D'Angelica M, Gonen M, Brennan MF, etal. Patterns of initial recurrencein completely resected gastric adenocarcinoma. Ann Surg. 2004;240:808-16.

12. Markar SR, Mikhail S, Malietzis G, et al. Influence of surgical resection of hepatic metastases from gastric adenocarcinoma on long-term survival: systematic review and pooled analysis. Ann Surg. 2016;263:1092-101.

13. Aizawa M, Nashimoto A, Yabusaki H, et al. Clinical benefit of surgical management for gastric cancer with synchronous liver metastasis. Hepatogastroenterology. 2014;61:1439-45.

14. Kinoshita T, Kinoshita T, Saiura A, et al. Multicentre analysis of long-term outcome after surgical resection for gastric cancer liver metastases. Br J Surg. 2015;102:102-7.

15. Kodera Y, Fujitani K, Fukushima N, et al. Surgical resection of hepatic metastasis from gastric cancer: a review and new recommendation in the Japanese gastric cancer treatment guidelines. Gastric Cancer. 2014;17:206-12.

16. DeMatteo RP, Lewis JJ, Leung D, et al. Two hundred gastrointestinal stromal tumors: recurrence patterns and prognostic factors for survival. Ann Surg. 2000;231:51-8.

17. Katz SC, DeMatteo RP. Gastrointestinal stromal tumors and leiomyosarcomas. JSurg Oncol. 2008;97:350-9.

18. Adam R, Chiche L, Aloia T, et al. Hepatic resection for noncolorectal nonendocrine liver metastases: analysis of 1,452 patients and development of a prognostic model. Ann Surg. 2006;244:524-35.

19. Seesing MF, Tielen R, van Hillegersberg R, et al. Resection of liver metastases in patients with gastrointestinal stromal tumors in the imatinib era: A nationwide retrospective study. Eur J Surg Oncol. 2016;42:1407-13.

20. Cananzi FC, Belgaumkar A, Lorenzi B, Mudan S. Liver surgery in the multidisciplinary management of gastrointestinal stromal tumour. AnzJ Surg. 2014;84:937-42.

21. DeMatteo RP, Maki RG, Singer S, et al. Results of tyrosine kinase inhibitor therapy followed by surgical resection for metastatic gastrointestinal stromal tumor. Ann Surg. 2007;245:347-52

22. AgarwalaSS, EggermontAMM, O’DayS,ZagerJS. Metastatic melanoma to theliver. Cancer. 2014;120:781-9.

23. Ryu SW, Saw R, Scolyer RA, et al. Liver resection for metastatic melanoma: equivalent survival for cutaneous and ocular primaries. J Surg Oncol. 2013;108:129-35.

24. Mariani P, Piperno-Neumann S, Servois V, et al. Surgical management of liver metastases from uveal melanoma: 16 years' experience at the Institut Curie. Eur J Surg Oncol. 2009;35:1192-7.

25. RyanDP,HongTS, Bardeesy N.Pancreaticadenocarcinoma. NEngl J Med. 2014;371:2140-1.

26. Rahib L, Smith BD, Aizenberg R, et al. Projecting cancer incidence and deaths to 2030: the unexpected burden of thyroid, liver, and pancreas cancers in the United States. Cancer Res. 2014;74:2913-21.

27. Yachida S, Jones S, Bozic I, et al. Distant metastasis occurs late during the genetic evolution of pancreatic cancer. Nature. 2010;467:1114-U26.
28. Tempero MA, Malafa MP, Behrman SW, et al. Pancreatic adenocarcinoma, version 2.2014: featured updates to the NCCN guidelines. J Natl Compr Canc Netw. 2014;12:1083-93.

29. Bellon E, Gebauer F, Tachezy M, et al. Pancreatic cancer and liver metastases: state of the art. Updates Surg. 2016;68:247-51.

30. Hackert T, Niesen W, Hinz U, et al. Radical surgery of oligometastatic pancreatic cancer. Eur J Surg Oncol. 2017;43:358-63.

31. Michalski CW, Erkan M, Huser N, et al. Resection of primary pancreatic cancer and liver metastasis: a systematic review. Dig Surg. 2008;25:473-80.

32. Tachezy M, Gebauer F, Janot M, et al. Synchronous resections of hepatic oligometastatic pancreatic cancer: disputing a principlein a time of safe pancreatic operations in a retrospective multicenter analysis. Surgery. 2016;160:136-44.

33. Thelen A, Jonas S, Benckert C, et al. Liver resection for metastases from renal cell carcinoma. World J Surg. 2007;31:802-7.

34. Staehler MD, Kruse J, Haseke N, et al. Liver resection for metastatic disease prolongssurvivalin renal cell carcinoma: 12 -year results from a retrospective comparative analysis. World J Urol. 2010;28:543-7.

35. Hatzaras I, Gleisner AL, Pulitano C, et al. A multi-institution analysis of outcomes ofliver-directed surgery for metastatic renal cell cancer. HPB (Oxford). 2012;14:532-8.

36. Neumann UP, Fotopoulou C, Schmeding M, et al. Clinical outcome of patients with advanced ovarian cancer after resection of liver metastases. Anticancer Res. 2012;32:4517-21.

37. Kamel SI, de Jong MC, Schulick RD, et al. The role of liver-directed surgery in patients with hepatic metastasis from a gynecologic primary carcinoma. World J Surg. 2011;35:1345-54.

38. Hahn TL, Jacobson L, Einhorn LH, et al. Hepatic resection of metastatic testicular carcinoma: a further update. Ann Surg Oncol. 1999;6:640-4.

39. Huang J, Li Q, Zheng Y, et al. Partial hepatectomy for liver metastases from nasopharyngeal carcinoma: a comparative study and review of the literature. BMC Cancer. 2014;14:818.

40. Djenic B, Duick D, Newell JO, Demeure MJ. Solitary liver metastasis from follicular variant papillary thyroid carcinoma: A case report and literature review. Int J Surg Case Rep. 2015;6C:146-9.

41. Modlin IM, Oberg K, Chung DC, et al. Gastroenteropancreatic neuroendocrine tumours. Lancet Oncol. 2008;9:61-72.

42. Pavel M, Baudin E, Couvelard A, et al. ENETS Consensus Guidelines for the management of patients with liver and other distant metastases from neuroendocrine neoplasms of foregut, midgut, hindgut, and unknown primary. Neuroendocrinology. 2012;95:157-76.

43. Roche A, Girish BV, de Baere T, et al. Trans-catheter arterial chemoembolization as first-line treatment for hepatic metastases from endocrine tumors. Eur Radiol. 2003;13:136-40.

44. Gupta S, Johnson MM, Murthy R, et al. Hepatic arterial embolization and chemoembolization for the treatment of patients with metastatic neuroendocrine tumors: variables affecting response rates and survival. Cancer. 2005;104:1590-602.

45. Lesurtel M, Nagorney DM, Mazzaferro V, et al. When should a liver resection be performed in patients with liver metastases from neuroendocrine tumours? A systematic review with practice recommendations. HPB (Oxford). 2015;17:17-22. 